\title{
Water security analysis of the Middle River supply system: a foreSIGHT application
}

\author{
Culley, S. ${ }^{\text {a }}$, Westra, S. a, Bennett, B. ${ }^{\text {a }}$, Maier, H.R. ${ }^{\text {a }}$, Newman, J. ${ }^{\text {, }}$, van der Linden, L. ${ }^{\text {b }}$ and Irvine, M. ${ }^{\text {b }}$ \\ ${ }^{a}$ University of Adelaide \\ ${ }^{b}$ South Australian Water Corporation \\ Email: sam.culley@adelaide.edu.au
}

\begin{abstract}
Scenario-neutral approaches have become the preferred method of interpreting climate driven impacts on natural and engineered systems when analysing the reliability of water resource systems facing climate change. Scenario-neutral approaches help deal with uncertainty and potential decision paralysis, by stress-testing systems across a range of plausible future conditions, instead of analysing systems under a narrower selection of climate projections. This results in better identification of failure modes and a richer elucidation of system behaviour. A case-study application of a methodology that supports the bottom-up approach — driven by a software tool 'foreSIGHT' - is presented here. Applied to the Middle River Reservoir system, the scenario-neutral approach adopted here follows three steps to: i) provide insights into system dynamics, ii) reveal modes of failure due to changes in climate, and iii) understand when intervention may be required given multiple lines of evidence.
\end{abstract}

The Middle River system (situated in Kangaroo Island, South Australia) comprises of a single reservoir with relatively small storage volume relative to the annual catchment inflows, resulting in large seasonal fluctuations in reservoir levels with regular filling in winter and drawdown over the dry season. The reservoir can hold approximately $200 \mathrm{ML}$ more than the mean annual demand (450 ML), which is highly variable year to year. The occurrence of draw down below 6 and $2 \mathrm{~m}$ levels were of interest to the stakeholders, as they broadly indicate, the need to augment the water supply due to a decrease in water quality (below $6 \mathrm{~m}$ ), and the reservoir emptying (below $2 \mathrm{~m}$ ). The reliability of the water supply system in 2030 was the focus of the analysis, as this aligns with opportunities to upgrade infrastructure.

Observed hydrological data for the Middle River catchment was analysed, and a coupled hydrological, water balance and demand model was used in order to determine a historical baseline of system performance over the period of 1961-2005. When modelling the system in response to the historical baseline as a benchmark of expected performance, it was found that in dry years the demand can be high enough to draw down the reservoir below $6 \mathrm{~m}$. The modelled probability of that failure under a historical climate was $\sim 2 \%$. It was also found that following this period of stationary climate there has been an observed increase in PET of $8.3 \%$, indicating that the system is already experiencing a 'changed' climate compared to the historical baseline.

A climate stress-test of the Middle River water supply system following the foreSIGHT framework demonstrated that the system is sensitive to annual changes in rainfall and PET. These metrics both influence supply and demand, with decreases in rainfall primarily affecting streamflow, and increases in PET increasing the demand placed on the system. In many of these failures, the reservoir had not filled the previous year, therefore providing little warning to enable water augmentation strategies to be put into place. Under more extreme changes in climate, the reservoir cannot fill multiple years in a row, leading to multi-year failures.

To establish an understanding of how the climate might have changed in 2030 relative to the historical baseline, multiple lines of evidence were considered including multiple sources of climate model projections for the year 2030, and the recent trend in observed PET. It was found using the scenario-neutral approach that in the climate conditions projected for the year 2030 the probability of failure of the reservoir dropping below $6 \mathrm{~m}$ could increase from $2 \%$ to $7-8 \%$. Further, the observed trend in PET suggests the probability of failure may have already increased to $5 \%$. This highlights a significant system sensitivity to relatively small changes in climate.

Keywords: Scenario-neutral climate change impact assessment, climate stress-test, water security 


\section{INTRODUCTION}

Scenario-neutral climate change impact assessments have emerged as a preferred method of describing the impact of climate change on a system, building on the traditional scenario-led approach (Brown et al., 2012; Culley et al., 2016; Poff et al., 2016; Prudhomme et al., 2010). This is primarily due to an increased ability to explore the vulnerability of a system beyond that of the narrow focus provided by climate projections (Brown and Wilby, 2012). This increased ability comes from the exploration of climate futures ranging well beyond climate projections and any other lines of evidence, exploring different modes of climate change. This allows for a better identification of failure modes and greater insight into the behaviour of the system.

Integral to the success of a scenario-neutral approach is therefore the ability to generate a wide ranging series of perturbed climate scenarios, as part of a climate stress-test. This has previously been a barrier to the uptake of scenario-neutral approaches, with a particular challenge involved in creating time series of hydroclimatic data for use in system modelling (Culley et al., 2019; Guo et al., 2018). As a means to overcome these challenges, an open source software tool called foreSIGHT has been developed (Bennett et al., 2021). This modelling tool works by first defining a series of climate statistics of a future scenario (referred to as climate attributes), and then using formal optimization techniques to identify the parameters of a stochastic weather generator that will provide the desired time series. With the ability to explore a wider range of changes in climate, the main focus of a scenario-neutral approach can then be developing an understanding of system behaviour, and gathering lines of evidence to understand system reliability. To date, the lines of evidence used have not been a strong focus of scenario-neutral impact assessments, with most studies using an ensemble of climate change projections for the system (Brown et al., 2012; Culley et al., 2016; Prudhomme et al., 2010).

This paper provides an application of the foreSIGHT software to a case study, demonstrating how the climate time series generation can be used as part of a scenario-neutral impact assessment, and using multiple lines of evidence to design the system stress-test. The paper introduces the Middle River case study in Section 2, detailing the catchment dynamics and demand under historical conditions. The scenario-neutral methodology is then described in Section 3, following the results of the stress-test and a discussion in Section 4. A conclusion is presented in Section 5 .

\section{CASE STUDY}

The Middle River water supply system, situated in Kangaroo Island, South Australia, supplies a combination of municipal, recreational and agricultural users. The catchment is located approximately $50 \mathrm{~km}$ west of Kingscote, and has an area of $101 \mathrm{~km}^{2}$. The system is supplied entirely from surface water obtained from this catchment, and comprises a single reservoir with a relatively small storage volume $(640 \mathrm{ML})$ relative to the annual catchment inflows $(7140 \mathrm{ML})$. This small storage to inflow ratio results in large seasonal fluctuations in reservoir levels with regular filling in winter and drawdown over the dry season. Demand patterns also vary seasonally, including significant year-to-year variations (greater than $50 \mathrm{ML}$ ).

A system model was developed for the purpose of simulating how the Middle River supply system would perform under the different climatic conditions used in this assessment. The system model was specifically developed to quantify the drawdown of the reservoir, and any shortfall in supply that might occur when drawdown exceeds operational thresholds. The system model comprises a catchment scale rainfall runoff model, a stochastic model of net customer demand, and a mass balance model of the Middle River Reservoir. The customer demand model was developed by combining a data driven model with a process-based model. The data driven model estimated demand based on its correlation with climate and economic variables (fourweek accumulated PET, the real price for water, and four-week accumulated rainfall). The process based model estimated the supply shortfall arising from the filling and emptying of farm dams and rainwater tanks. The model also includes uncertainty associated with the variability in annual demand, which is modelled by an auto-regressive AR(1) component to the model.

The two performance measures for the Middle River reservoir are:

- Reliability above $6 \mathrm{~m}$ at the annual scale (chance that the reservoir level drops below $6 \mathrm{~m}$ ). This represents a point at which the water supply may need to be augmented due to the quality of the remaining water in the reservoir.

- $\quad$ Reliability above $2 \mathrm{~m}$ at the annual scale (chance that the reservoir level drops below $2 \mathrm{~m}$ ). Below this point is dead storage, and so the reservoir cannot supply any water.

The reliability of the Middle River system by the year 2030 is of interest to the stakeholders, given the alignment with a regulatory planning period. 


\section{METHODOLOGY}

This paper applies a scenario-neutral climate impact assessment approach (Brown et al., 2012; Culley et al., 2016; Poff et al., 2016; Prudhomme et al., 2010) to perform a reliability assessment of the existing Middle River system. The first step involves defining and understanding the system and how it performs under historical climate conditions (Section 3.1). The second step is to stress-test the defined system and identify the climate conditions under which it will fail. This process is implemented using the foreSIGHT software tool (Bennett et al., 2021) and its associated framework (Section 3.2). The third step is to analyse climate projections and other lines of evidence to determine the reliability of the system, by considering the likelihood and possible timing of failure occurring (Section 3.3).

\subsection{Description of historical system performance}

Before understanding the reliability of the system, it is necessary to establish a baseline of system performance. This baseline will then allow stress-testing under historical climate conditions to indicate how system performance will change, and also provide some insight in to the system mechanics that will allow the dimensions of the climate stress-test to be put forward in Section 3.2 (Culley et al., 2021).

Firstly, the system model described in Section 2 is used to simulate a historical baseline period of observed climate. As the demand model is stochastic, the historical baseline will be simulated 100 times. In addition to establishing a baseline of system performance, this provides an opportunity to work with end users to ensure the modelled historical baseline is representative of actual system behaviour. It also enables a more in-depth exploration of how the system has responded to historical climate. The historical baseline used in this assessment is the period from 1961-2005. This was taken as the baseline as this time period aligns with the hindcast used in climate projection model databases such as SAClimateReady (Charles and Fu, 2015) and Climate Change in Australia (CSIRO and Bureau of Meteorology, 2015), and combines two periods regularly used by the international panel on climate change (IPCC); 1961-1990 and 1986-2005. The reliability of the reservoir level staying above 6 and $2 \mathrm{~m}$ is calculated for this period.

\subsection{Stress-test the system}

To identify which changes in climate most influence the performance of the Middle River system, it is necessary to stress-test the system under a broad range of climate attributes. This approach follows the approach detailed in the foreSIGHT framework, which is to first define a series of climate attributes to be perturbed (Section 3.2.1), and then use stochastic weather generators to generate the corresponding time series in order to simulate the system response (Section 3.2.2).

\subsubsection{Defining the stress-test}

First, a set of climate attributes that are likely to be significant to the Middle River system is proposed. For rainfall, the annual total and the number of wet days in the year are included, as well as the $99^{\text {th }}$ percentile rainfall each year to cater to relatively heavy rainfall events. Given the impact consecutive dry days can have on streamflow, the annual average dry spell duration is also included. Due to the strong rainfall seasonality, each seasonal total is included, as well as a measure of the seasonality (i.e. the amplitude of the monthly rainfall totals). Finally, given the annual variability of rainfall totals over the 45 year historical baseline, the minimum total annual rainfall volume over a 45 year time period, and the standard deviation of annual totals, are included. For PET, the annual total and the same measure of seasonality are used.

Multiple lines of evidence are then considered (see Table 1) to understand likely changes in climate the system will experience over the period of interest. As 2030 is the end of the planning period, the SA Climate Ready projections for each attribute are summarised for that window under RCP8.5. Given there are 15 different climate models, the mean projected change is shown, as well as the $10^{\text {th }}$ and $90^{\text {th }}$ percentile of projected changes. Also included in Table 1 are the Climate Change in Australia projections for annual rainfall and PET. These projections are a broad summary of the entire southern Australian region, and so no specific summaries are given for more complex climate attributes. The observed changes in PET from the SILO record are also included. This does not represent a projection of climate conditions in 2030 such as those derived from the climate models, but is important to include to provide a sense of plausible changes in PET based on historical patterns of variability.

In this study, these multiple lines of evidence considered are used to determine the bounds of the stress-test. The bounds are selected to extend beyond the range of all data points, acknowledging the regions of change that are more likely to occur for the time period of interest, while also accounting for the large uncertainty in climate projections. The stress-test is then defined by perturbing changes in each climate attribute in Table 1 
Culley et al., Water security analysis of the middle river supply system: a foreSIGHT application

in steps of 5\%. The attributes are perturbed one-at-a time to identify those with the greatest influence on system performance, which will then be used in the reliability analysis.

Table 1. Multiple lines of evidence of climate change, and resulting stress-test bounds on key attributes

\begin{tabular}{|c|c|c|c|c|c|c|c|}
\hline \multirow[b]{3}{*}{ Attribute } & \multicolumn{5}{|c|}{ Lines of evidence (\% change from historical baseline) } & \multirow{2}{*}{\multicolumn{2}{|c|}{$\begin{array}{l}\text { Stress-Test } \\
\text { Bounds }\end{array}$}} \\
\hline & \multicolumn{3}{|c|}{ SA Climate Ready } & \multirow{2}{*}{$\begin{array}{c}\text { Climate Change } \\
\text { in Australia }\end{array}$} & \multirow{2}{*}{$\begin{array}{c}\text { Observed } \\
\text { SILO record }\end{array}$} & & \\
\hline & $\begin{array}{l}2030 \\
\text { low }\end{array}$ & $\begin{array}{l}2030 \\
\text { mean }\end{array}$ & $\begin{array}{c}2030 \\
\text { upper }\end{array}$ & & & Lower & Upper \\
\hline Total annual rainfall & $-13.2 \%$ & $-6.9 \%$ & $-1.9 \%$ & $-10 \%$ & & $-20 \%$ & $0 \%$ \\
\hline Annual heavy rainfall & $-6.9 \%$ & $-2.5 \%$ & $+1.7 \%$ & & & $-10 \%$ & $+5 \%$ \\
\hline Rainfall seasonality & $-7.2 \%$ & $-1.7 \%$ & $+4.5 \%$ & & & $-10 \%$ & $+10 \%$ \\
\hline Annual number of wet days & $-10.2 \%$ & $-5.8 \%$ & $-2.4 \%$ & & & $-15 \%$ & $+5 \%$ \\
\hline $\begin{array}{l}\text { Annual average dry spell } \\
\text { duration }\end{array}$ & +1.7 & $+6.2 \%$ & $+10.7 \%$ & & & $0 \%$ & $+15 \%$ \\
\hline Annual total PET & $+4.9 \%$ & $+6.8 \%$ & $+9.3 \%$ & $+3 \%$ & $+8.3 \%$ & $0 \%$ & $+10 \%$ \\
\hline PET seasonality & $-1.5 \%$ & $+3.6 \%$ & $+12.9 \%$ & & & $95 \%$ & $+15 \%$ \\
\hline
\end{tabular}

\subsection{2 foreSIGHT methods used}

Two stochastic weather generators from foreSIGHT are used to generate the climate time series: one for rainfall and one for PET. Throughout the analysis, 50 replicates with the same length as the historical baseline are generated. The rainfall weather generator uses the WGEN model (Richardson and Wright, 1984). A $1^{\text {st }}$ order Markov chain generates the wet day/dry day sequence, and a gamma distribution is then used to sample rainfall amounts. The four parameters that control the WGEN model (probability wet-dry and probability dry-dry for Markov chain, and shape and rate parameters for gamma distribution) vary throughout the year, each fitted to a harmonic. Using a harmonic to vary the parameters allows for seasonality in the generated rainfall, without adding too much complexity. Monthly scaling factors with mean 1 and range 0.8 are then applied over the whole time series, to increase the standard deviation of the monthly totals to better reflect historical rainfall statistics. The PET weather generator uses two harmonics to set the mean and standard deviation of PET through the year. Monthly samples of the means and standard deviation are then taken, with PET values generated by adding the standard deviation multiplied by a residual to the mean. An additional parameter controls the lag-1 correlation used to generate the residuals (Richardson and Wright, 1984). PET is generated independently of it being a wet day or a dry day.

\subsection{Determine system reliability}

To understand the reliability of the Middle River system for the planning horizon of the next ten years, the system understanding and possibilities of failure identified in Section 4 need to be compared with climate model projections and other lines of evidence to understand how likely any failures are to occur. The two most influential attributes identified in the previous step of the methodology are used to generate a scenario-neutral space detailing system response to changes in combinations of both attributes. The multiple lines of evidence are then overlaid in order to determine plausible future performance of the Middle River reservoir by 2030.

\section{RESULTS AND DISCUSSION}

\subsection{Historical baseline of the Middle River system}

In the 100 baseline simulations of the Middle River reservoir demand model in response to historical climate (i.e. 4500 years total) the reservoir drew down below $6 \mathrm{~m} 14$ times (Figure 1). This occurs most frequently in the second year, which has a median drawdown of $6.9 \mathrm{~m}$, making the reliability sensitive to the demand in this year. This corresponds to an annual $0.4 \%$ chance of the reservoir falling below $6 \mathrm{~m}$. The lowest recorded level was $5.0 \mathrm{~m}$, and so the other performance threshold of $2 \mathrm{~m}$ was not exceeded. Although the reliability above 6 $\mathrm{m}$ is close to 1, it is important to note that given the climate conditions of 1961-2005, a very high demand year can draw the reservoir down below $6 \mathrm{~m}$ even if the reservoir fills during the previous wet season. 


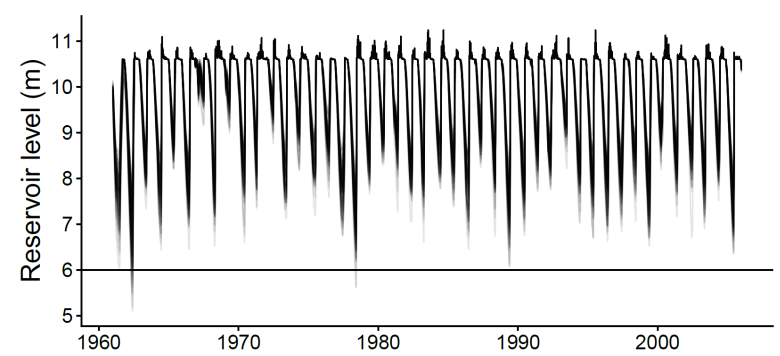

Figure 1. System performance over the historical baseline used

\subsection{Stress-test of the middle river system}

The one at a time stress-test perturbs each of the attributes in Table 1, according to the bounds, at steps of 5\% change from historical levels. The fifty replicates used to perturb the historical baseline provide a total of 2250 years of simulation. The annual chance of the reservoir level being below both $2 \mathrm{~m}$ and $6 \mathrm{~m}$ is shown on the left axes, with the corresponding reliability on the right. For each step change, the chance of failure is calculated as the number of years the reservoir drops below each threshold level, out of the full 2250 years of record. On both Figure 2 and Figure 3, the mean projected change for 2030 for each of the 15 SA Climate Ready models is also shown, to give an indication of how plausible the step changes are.

The five one-at-a-time tests for rainfall (Figure 2) show how the reliability of the reservoir is affected by different modes of climate change. The one-at-a-time tests show that the most significant change in reliability is when the total annual rainfall decreases. With a $20 \%$ decrease in rainfall, the annual chance of the reservoir dropping below $6 \mathrm{~m}$ is $12 \%$. This is primarily due to a reduction in inflows to the reservoir, and when the reservoir does not fill during winter as often there are more failures the following year.
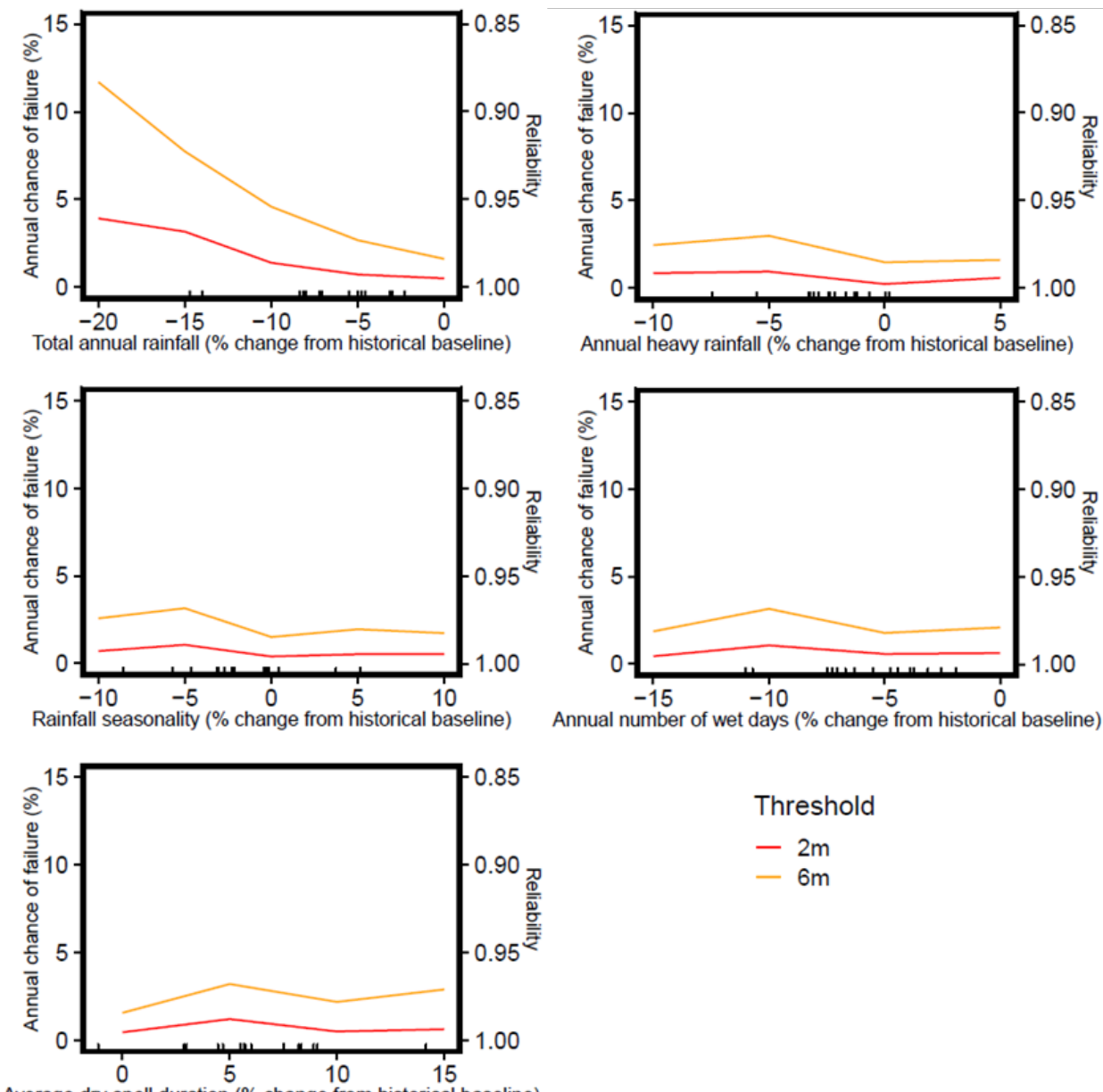

Figure 2. A climate stress-test of rainfall attributes against the system performance metrics 
All other changes have second order effects, as with the annual rainfall held constant and broadly the same seasonal pattern of rainfall, annual streamflow can only change slightly as a result of different wet day occurrences. While it is not as clearly seen from the reliabilities, of the remaining attributes after annual rainfall, the largest impact on demand was average dry spell durations and the largest impact on inflow was the rainfall seasonality.

The PET one at a time tests (Figure 3) show that the annual changes are not likely to have as strong an effect on performance as rainfall. The annual chance of dropping below $6 \mathrm{~m}$ has increased to $5 \%$, which is similar to the effect of a $10 \%$ decrease in rainfall. The increasing seasonality of PET (i.e. summer PET increasing and winter PET decreasing) has a much smaller effect on the reliability of the reservoir, when annual PET is held constant. The average annual demand still increases under these scenarios, but changes in inflows are quite small.
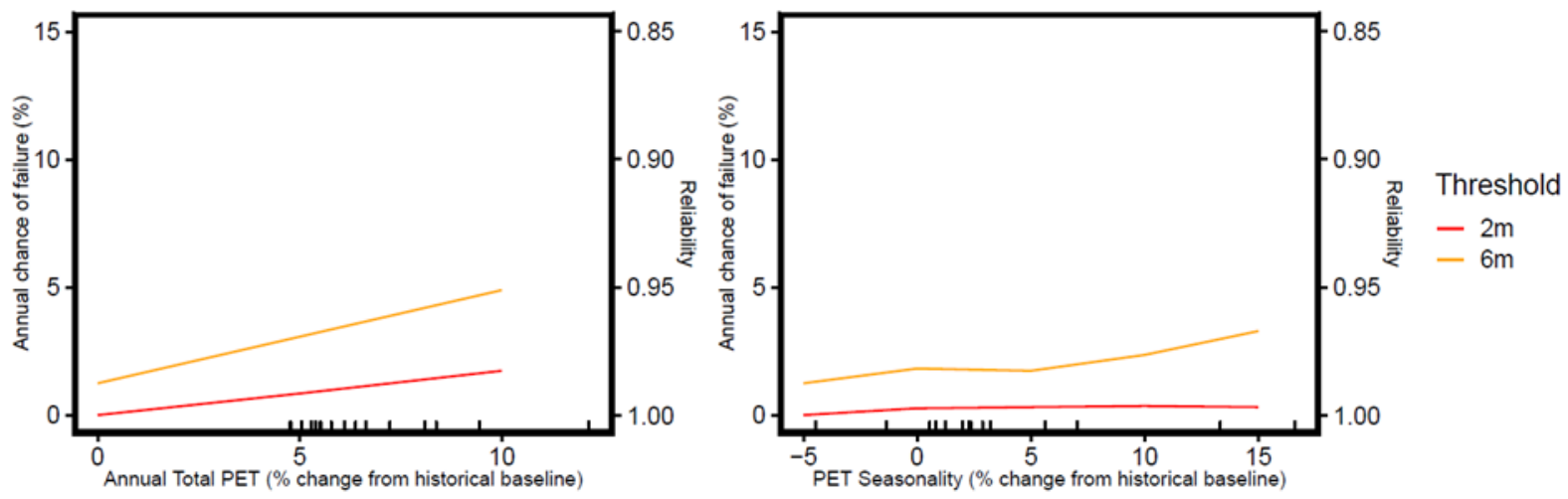

Figure 3. A climate stress-test of PET attributes against the system performance metrics

\subsection{Reliability of the middle river system}

To determine plausible future climate conditions, the lines of evidence in Table 1 are overlaid on top of the scenario-neutral stress-test (Figure 4). The fifteen climate model projections from RCP8.5 are shown (grey), as well as the mean of the ensemble (orange) and the 2006-2019 PET compared to the historical baseline (red). By considering where in the scenario-neutral space the alternative lines of evidence are located, the performance as a result of those climate conditions can be inferred. For example, both the mean SA Climate Ready projections and the SILO data suggest the chance of failure below $6 \mathrm{~m}$ could be $\sim 5 \%$, and the chance of failure below $2 \mathrm{~m}$ could be 1-2\%. Twelve of the climate models are clustered around the mean, but there are four models that lie further away, suggesting that the risk could increase up to $\sim 12 \%$ and $\sim 4.5 \%$ for $6 \mathrm{~m}$ and 2 $\mathrm{m}$, respectively.
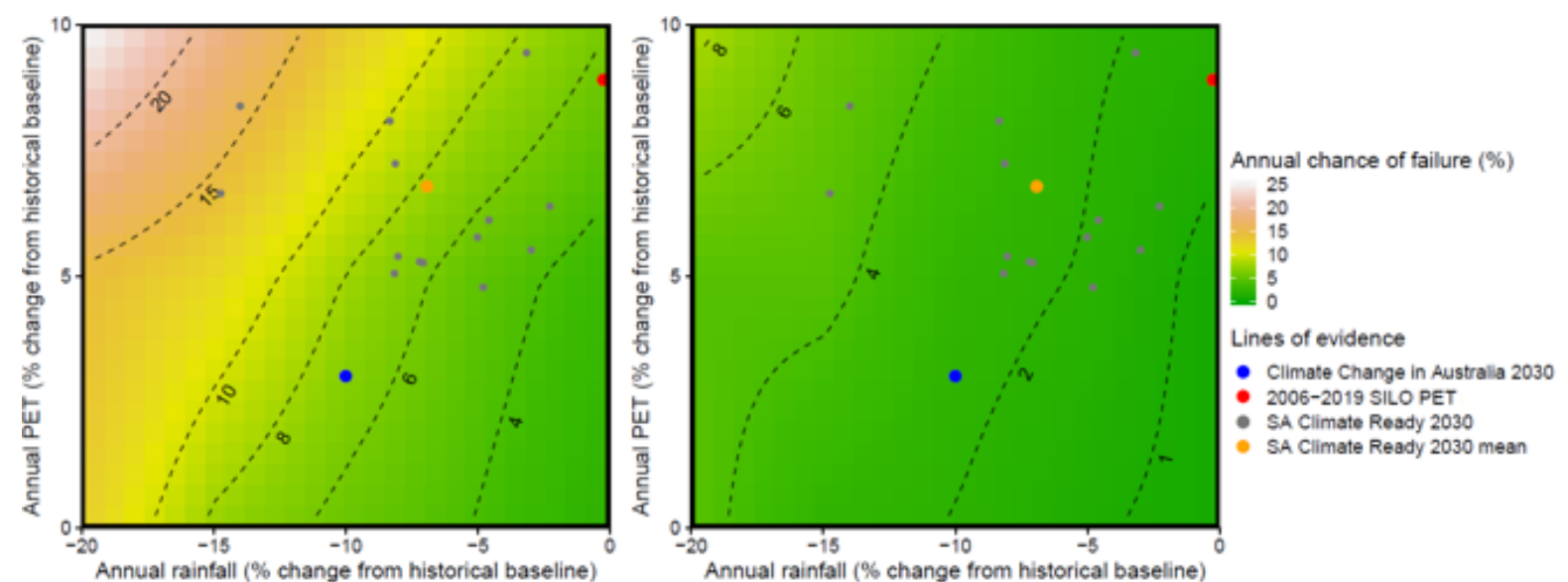

Figure 4. Scenario-neutral impact assessment of the Middle River reservoir for 2030. Annual chances of failure are shown for $6 \mathrm{~m}$ (left) and $2 \mathrm{~m}$ (right).

In the majority of cases where the storage level fell below $6 \mathrm{~m}$ in the stochastic recreation of the historical baseline, the reservoir did not fill in the previous year, allowing $\sim 6$ months warning before a drawdown below $6 \mathrm{~m}$ occurs. This provides an opportunity for deploying short-term adaptation measures like preparing pumping 
Culley et al., Water security analysis of the middle river supply system: a foreSIGHT application

from nearby water supplies or the treatment of the reservoir storage to allow supply to occur as the level drops below $6 \mathrm{~m}$. However, there is also a non-zero chance that if failure occurs, there are no warnings from the previous year (like those in response to the observed historical baseline (Section 4.1).

\section{CONCLUSIONS}

This paper presented a scenario-neutral climate impact assessment of the Middle River water supply system on Kangaroo Island, South Australia, in order to determine the water security in the year 2030. The impact assessment first defined the historical performance over a stationary historical climate baseline, identifying that in dry years the reservoir can draw down below acceptable levels of performance, with a modelled probability of $2 \%$. After establishing a performance baseline, the foreSIGHT tool and framework were used to perform a climate stress-test of the system, identifying annual changes in precipitation and PET to be the primary drivers of system performance. A scenario-neutral space was developed for these two climate attributes, and multiple lines of evidence were overlaid to identify the system reliability in the year 2030. Crucially, an observed increase in PET for the region also indicated that the current climate has moved past the historical baseline, and performance could degrade to a $5 \%$ chance of failure. It was also found that the annual change of failure in response to median climate change projections for the region could increase to $6-7 \%$ by the year 2030 . The results of this analysis demonstrate both the utility of the foreSIGHT tool in system planning, and the importance of considering multiple lines of evidence in scenario-neutral impact assessments.

\section{REFERENCES}

Bennett, B., Devanand, A., Culley, S., Westra, S., Guo, D., Maier, H.R., 2021. A modelling framework and Rpackage for evaluating system performance under hydroclimate variability and change. Environmental Modelling \& Software 139104999.

Brown, C., Ghile, Y., Laverty, M., Li, K., 2012. Decision scaling: Linking bottom-up vulnerability analysis with climate projections in the water sector. Water Resources Research 48(9) W09537.

Brown, C., Wilby, R.L., 2012. An alternate approach to assessing climate risks. Eos, Transactions American Geophysical Union 93(41) 401-402.

Charles, S., Fu, G., 2015. Statistically downscaled climate change projections for South Australia. Adelaide, South Australia.

CSIRO, Bureau of Meteorology, 2015. Climate change in Australia information for Australia's natural resource management regions: Technical report. CSIRO and Bureau of Meteorology Australia.

Culley, S., Bennett, B., Westra, S., Maier, H.R., 2019. Generating realistic perturbed hydrometeorological time series to inform scenario-neutral climate impact assessments. Journal of Hydrology 576 111-122.

Culley, S., Maier, H.R., Westra, S., Bennett, B., 2021. Identifying critical climate conditions for use in scenarioneutral climate impact assessments. Environmental Modelling \& Software 136104948.

Culley, S., Noble, S., Yates, A., Timbs, M., Westra, S., Maier, H., Giuliani, M., Castelletti, A., 2016. A bottomup approach to identifying the maximum operational adaptive capacity of water resource systems to a changing climate. Water Resources Research 52(9) 6751-6768.

Guo, D., Westra, S., Maier, H.R., 2018. An inverse approach to perturb historical rainfall data for scenarioneutral climate impact studies. Journal of Hydrology 556 877-890.

Poff, N.L., Brown, C.M., Grantham, T.E., Matthews, J.H., Palmer, M.A., Spence, C.M., Wilby, R.L., Haasnoot, M., Mendoza, G.F., Dominique, K.C., Baeza, A., 2016. Sustainable water management under future uncertainty with eco-engineering decision scaling. Nature Clim. Change 6(1) 25-34.

Prudhomme, C., Wilby, R.L., Crooks, S., Kay, A.L., Reynard, N.S., 2010. Scenario-neutral approach to climate change impact studies: Application to flood risk. Journal of Hydrology 390(3-4) 198-209.

Richardson, C.W., Wright, D.A., 1984. WGEN: A model for generating daily weather variables. 